\title{
Democracy and polarization
}

\section{Comments on Aikin and Talisse's} Political argument in a polarized age

\author{
Anand VAIDYA*
}

\section{INTRODUCTION}

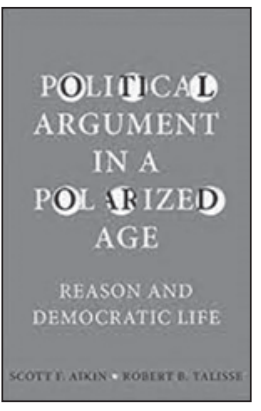

Political argument in a polarized age: reason and democratic life (PAPA, hereafter) by Scott Aikin and Robert Talisse (the authors, bereafter) is a timely and engaging study of the state of American democracy. It is not just an academic piece, but an excellent instance of public philosophy where the public plays a guiding role in the construction of the philosophy. It is the kind of public philosophy I have elsewhere called, the public-to-philosophy direction, as opposed to the philosophy-to-public direction. ${ }^{1}$

I am, largely, in agreement with the views forwarded by the authors. Nevertheless, Iwant toengage the topicofcriticalthinking in democracy by deploying the perspectival epistemology of Jain philosophy in order to provide a wider set of critical questions, which lead to a more complete picture. $^{2}$

In what follows I will first pull on a few threads that I would like the authors to discuss in more detail. Here my aim is to provide them with an opportunity to either clarify their view or expand it to cover something that was

${ }^{1}$ See Vaidya, 2015, for discussion of two types of public philosophy.

${ }^{2}$ See Vaidya, 2018, for discussion of Jain contributions to critical thinking education.

* Professor of Philosophy at San Jose State University and Director of the Center for Comparative Philosophy, USA. E-mail: anand.vaidya@sjsu.edu. 
not considered in PAPA. However, I will also argue that the picture of political argumentation presented by the authors is incomplete in so far as it presents the phenomenon as a rational enterprise at the cost of leaving out how status negotiation is part of political argumentation. Throughout I will be laying the foundation for my closing question: should some forms of polarization rationally entice participants in a democracy to form two new democracies where there is greater alignment within each of the new democracies?

\section{WHAT IS DEMOCRACY?}

On my reading of PAPA, the authors advance the disagreement thesis about democracy:

We understand democracy to be the proposal that a stable and decent political order can be sustained by equal citizens who nonetheless disagree, often sharply, about the precise shape their collective life should take (Aikin \& Talisse, 2020: 2).

[D] emocracy is a system of politics based in the premise that equal citizens are bound to disagree, sometimes profoundly, about what is good. Such disagreement is, after all, the upshot of our political equality. That's an important thing to remember about this ideal notion of democracy - central to it, even in the ideal form, is the notion that free and equal citizens will have significant disagreements and, because they are equals, they can't just boss each other around (Aikin \& Talisse, 2020: 13).

The disagreement thesis holds that disagreement is both essential to proper democratic functioning and central to democratic practices. However, there are two readings of the disagreement thesis, which are not separated clearly.

On the one hand, the disagreement thesis could be read as suggesting that political equals in a democracy should disagree with each other, regardless of what they believe, because the best way to find out what is good for everyone is for there to be reasoned exchanges between individuals, experts or not, for the purposes of epistemically evaluating various options. The disagreement thesis could also be read as suggesting that political equals in a democracy will disagree about how to live collectively because it is highly improbable that individuals will share a common enough conception of what is morally best for everyone - at best our moral outlooks will only partially agree.

The epistemic reading is consistent with the idea that a democracy can be properly run when all citizens hold the same basic moral position. The moral reading is inconsistent with this view. One might ask the authors: could there exist a democracy where there is only an epistemic reason for disagreement because there is total moral overlap on what the good life is? And is this kind 
of democracy healthier than one in which there is both moral and epistemic disagreement?

\section{CAN WE ARGUE WELL?}

The authors argue that (Aikin \& Talisse, 2020: 25-26):

1) Disagreement is central to democracy.

2) In order to fulfill our duties as democratic citizens, we need to engage in civil political disagreement.

3) Civil political disagreement is built on proper argumentation - well-ordered rational exchange between interlocutors.

4) Proper argumentation is a collective enterprise that requires interlocutors to assess each other's argumentative performance.

5) Therefore, disagreeing well involves an earnest attempt to diagnose the disagreement, to find its root, to explain why dissensus persists.

They further point to the need for a shared vocabulary for assessing the variety of positions that are in play. However, this brings up a couple of questions. First, there is the problem of who and where the shared common vocabulary should come from. Who gets to determine what the correct vocabulary should be? Second, there is the problem of what standards need to be met for one's challenges to be taken seriously. If the idea is that arguing well requires training, what distinguishes competence from non-competence and who sets and monitors those standards?

Take, for example, critical thinking courses taught at universities around America. The standard model traces back to Plato and Aristotle, and does little to consider the contributions to critical thinking made by non-Western thinkers. One example where we can see the problem arise is in the use of the qualifier "evidence-based". The term "evidence-based" often contrasts with "non-evidence based", so as to suggest that those that do not use the qualifier "evidence-based" have no evidence for the claims they make. What is more salient is that the qualifier "evidence-based" aims to pick out evidence that is consistent with and appropriate within the context of science. There are epistemic cultures which either (i) recognize different sources of evidence, or (ii) treat non-scientific sources as providing some way of knowing about the world. This leads to the question how can we argue well when we don't agree on the vocabulary for argumentation because we don't share a sufficiently similar epistemic culture? That is, how can we build a shared vocabulary for argumentation if we don't have a common enough epistemic culture? 


\section{IS POLARIZATION A PROBLEM THAT CAN BE OVERCOME?}

The authors provide a useful distinction between belief and political polarization:

As belief polarization leads us to regard our political rivals as increasingly benighted, irrational, and unreasonable, we become more and more inclined to distrust, dislike, and resent those whom we regard as our opponents. We thus become ever more involved only with our political allies, and this in turn contributes further to belief polarization. Our political alliances thereby become more tightly knit and exclusionary. Consequently, political parties and their leaders are incentivized to punctuate (and overstate) their policy and platform differences. All this occurs within a self-perpetuating, spiraling dynamic that intensifies civic divisions and interpartisan animosity. That is, belief polarization sets in motion a broader dynamic that not only codifies political polarization, but also erodes our capacity for civil political disagreement, and thus democracy (Aikin \& Talisse, 2020: 39).

If belief polarization is a natural human tendency and it leads to political polarization which in turn leads to tribalism in a way that makes us unable to see those outside our tribe as political equals, we might wonder whether it is madness to bind citizens to each other when polarization is bound to occur. Consider an analogy with marriage.

A marriage binds two individuals together as equals in a conversation about how best to live their lives together. Democracy binds a plurality of citizens together as equals in a conversation over how best to live together. However, in a marriage we often choose our partner on the basis of common traits that would likely lead to a happy marriage. In a democracy, most of us, do not choose who the equals are that we are bound to. In a marriage there is the option of deciding that there are irreconcilable differences or deep disagreements, and on the basis of the differences and disagreement, the parties would be better served if they did not continue their union. In a democracy we might wonder: what real options are available for those that decide that there are irreconcilable differences between them and other citizens? In section 10, I will return to this analogy in order to draw out the question of whether certain kinds of polarization can rationally lead one to believe that, while democracy is a good form of government, they are in an unhealthy democracy because the participants have irreconcilable differences, in much the same way that one can believe that marriage is a good social institution, while believing that certain marriages are unhealthy because the participants have irreconcilable differences. 


\section{CAN WE OVERCOME POLITICAL IGNORANCE?}

As the authors note, one of the central problems facing democracies is the problem of political ignorance:

Ever since Plato, the widespread ignorance of the citizens has functioned as a central argument against democracy. And so, we must ask: why should equal political power be apportioned to citizens who are ignorant? Or, to formulate the same thought in a different way, why must you regard those whom you believe to be fundamentally mistaken about the most important political issues as your political equals? Shouldn't their incompetence disqualify them from democratic citizenship? (Aikin \& Talisse, 2020: 43)

However, on my view the problem of political ignorance presented here is incomplete.

On the one hand, it is easy to see why we should see citizens with whom we disagree with on the most fundamental issues as our political equals. The mere fact that we are members of the same democracy grounds our political equality. Moreover, we should not infer from the fact that two people are unequally informed that they are not political equals. Rather, the correct inference is that that they are not epistemic peers with respect to political matters. When a person senses that another is not sufficiently informed, they ought to think of the individual as, perhaps, negligent, rather than as politically unequal. Epistemic inequality is not sufficient for political inequality.

On the other hand, the authors suggest that epistemology and critical thinking can be helpful in diagnosing political ignorance in a more nuanced manner. Consider the case of Ann and Bruce as provided by the authors.

- Ann believes that the president directly controls the price of gasoline because the otherwise credible news channel she regularly watches constantly features reports that blame the president for the price of gas.

- Bruce believes that the president directly controls the price of gasoline despite being regularly exposed to sources of correction and evidence sufficient to require him to revise his belief.

Ann and Bruce both believe something false: the president directly controls the price of gasoline. However, what it takes to make Ann politically informed is different from what it takes to make Bruce politically informed. Ann, for example, is not epistemically irresponsible, and perhaps not blameworthy, she is subjectively justified in her belief. Bruce, on the other hand, is epistemically irresponsible. Ann perhaps needs to just check some other news sources or discuss the issue with friends. Bruce, in contrast, needs a critical thinking 
course. The authors are right to point to the fact that political ignorance can be differentiated, each requiring different methods for correction. I agree that theoretical and applied epistemology as well as critical thinking can do a lot. But epistemology and critical thinking might not go far enough. There is a threshold. Just because epistemology and critical thinking can make us better in our public engagements, it will not follow that it will make us collectively good enough. Even if it can, a concrete procedure needs to be detailed that shows how it can. Let's look at two topics not discussed by the authors: deep fakes and bounded rational decision-making.

Consider the problem of deep fakes. Deep fakes, unlike ordinary fake news, are put together so well that the ordinary citizen cannot determine that it is fake. Given that advances in technology will allow for the easy creation and dissemination of deep-fakes (including deep-fake audio and video files), one would be justified in being skeptical about most sources of information, particularly in the domain of politics. One might end up being epistemically paralyzed with respect to belief formation about political matters because it is too difficult to determine who to trust. Rather than individually attempting to form a justified belief, they might end up outsourcing a lot of their critical thinking to others that they consider to be epistemic superiors.

The problem of deep fakes can be compounded by adding, as we ought to, the confines of time. The real question of political ignorance ought to be: for the average citizen, who is employed 40 hours a week, has a family, can only spend some time watching normal news outlets, and has very limited free time to counter check news sources, is it plausible that they will be able to sort through and counter check information, such that they should feel confident enough to make an epistemically well-informed decision about, for example, which candidate to vote for?

Given these constraints, it might be far simpler to take on a political identity, such as belonging to a particular party, which would allow for the partial outsourcing of critical thinking to the pundits who are members of their party. Moreover, if we don't have time to engage in political discussions, and cannot even agree on the facts, why shouldn't we simply join a party where other members take on the epistemic burden of determining the correct views to hold? What does it take to be epistemically responsible with respect to critical thinking in a democracy where we have to make decisions in time and based on possibly tainted information? ${ }^{3}$

\footnotetext{
${ }^{3}$ See Huemer, 2005, vs. Vaidya, 2013, for debate over whether individual critical thinking is epistemically responsible when it comes to important issues that are decided by voting in a democratic processes. Huemer holds that critical thinking is not epistemically responsible, and Vaidya argues that it is.
} 


\section{THREE DISTINCTIONS CONCERNING ARGUMENTATION}

One of the main points that the authors aim to make about political argument in a polarized age is that there is a lot of simulated rather than real argumentation going on. Simulated argument occurs when interlocutors put on the show of an argument, but don't really engage each other. They don't consider real objections, and do not aim to address each other in a real and meaningful way.

The authors distinguish between rhetorical and epistemic views of argumentation. The rhetorical view holds that the only purpose of argument is to convince others, and because of that an argument is successful to the degree that it brings about greater consensus. The epistemic view of argument holds that we argue in order to find out what is best supported by the evidence and reasons. The idea is that in an argumentative exchange we can come to see more fully what reasons and evidence there are, and what positions are best to occupy. One contrast between the two views concerns how civility fits into argumentation. The epistemic view locates civility in seeing others as partners in the common pursuit of believing based on what the best evidence leads us to. The rhetorical view locates civility in one individual taking another individual as they are with their beliefs in a rational attempt to move them to their own view. There is no argumentative success on the rhetorical view, if there is no increase in consensus, while on the epistemic view there can be success even if no one is moved to a different side, perhaps because greater understanding of all positions is improved by the exchange.

The authors hold that the rhetorical mindset of argumentation involves viewing one's interlocutor as a "patient", while the epistemic mindset involves viewing the interlocutor as a "partner". Thus, the interlocuter engaged in rhetorical debate is keen to point out and diagnose what is wrong with the opposing point of view, without the need to acknowledge and identify areas of agreement. In the partner model the point of arguing is for each person to work with their interlocuter in order to get a better sense of their own position and to determine what to believe. In the partner model neither person aims only to diagnose what is wrong with the other person. Rather, they also seek to learn what might be wrong with their own position in a joint search for what to believe. While the distinction is important, the authors make it seem like you can only have one, they seem to imply, for example, that the patient and the partner roles are fixed rather than fluid and context-dependent. I wonder if there is another way to see the distinction.

Consider the distinction I favor between positioned and discovery-argumentation, which while similar to rhetorical and epistemic views of argumentation, allows for serial switching between the two roles. Positioned-argumentation occurs when an individual or group argues to promote a position and defend it against known attacks. In a sense positioned-argumentation is 
simulated argumentation because it is the performance of the reasons that go into a position as well as a defense of it against known attacks - it is a form of rhetorical argumentation in that it seeks to convince others of the position. However, it need not be wedded to the idea that the only success is greater consensus. Discovery-argumentation, by contrast, occurs when individuals or groups argue with one another in order to discover what position to take or how their position plays out against the views of others. In discovery-argumentation one might not know how others will respond to their argument. Participants in discovery-argumentation view their role as one that involves enriching the epistemic community in addition to advancing a specific position. The difference between the rhetorical / epistemic distinction and the positioned / discovery distinction is that the former appears to be binary while the latter is not.

To illustrate the possiblity of serial switching between the two modes in the latter distinction consider the following. One might first engage in discovery-argumentation with a diverse and robust group of thinkers in order to figure out what to defend. But once fixed on a position, one might then engage in positioned-argumentation, so as to expose the position and defend it against attacks that were brought out in the discovery phase. However, that exercise in positioned-argumentation might then lead to a new counter-position that was not brought out in the earlier discovery phase. This discovery within the exercise of positioned-argumentation might then lead to a revision followed by a further defense of the new updated position. While I prefer an epistemic-partner view of argumentation over that of a rhetorical-patient view of argumentation, I wonder if there really is no legitimate purpose that rhetorical argumentation can serve in political argumentation? It is not clear that the authors hold that there is no legitimate purpose, but advocating for the epistemic-partner view as appropriate for the culture of political argumentation makes it seem as if rhetorical-patient argumentation is not appropriate. Could certain disagreements require a rhetorical rather than an epistemic stance? If the beliefs of one group lead to the view that members of the other group are not their political equal, why couldn't the latter group's response take on a rhetorical-patient voicing, for what partnership can one be in with someone who does not see them as a political equal?

\section{DEEP DISAGREEMENT AND THE POSSIBILITY OF COMMUNICATION}

The authors note that unlike normal disagreements, where participants in the exchange share enough common ground to resolve the disagreement, deep disagreements have some good news and bad news. The bad news is clear: 
a way to argue with someone with whom you do not share background commitments sufficient to resolve the issue. The arguments you give will employ premises that they reject, and arguments they give you will have premises that will strike you as unacceptable or irrelevant. In such cases, it seems as if rational discourse cannot prevail (Aikin \& Talisse, 2020: 80).

But, deep disagreements also provide some good news. Here is their argument. ${ }^{4}$

1) (A) robust intellectual freedom and (B) a lower probability that one's beliefs are based on social conditioning alone, as opposed to reason and evidence, are epistemic goods worth having.

2) Where there are only normal disagreements, it is more likely to find the opposite of (A) and (B).

3) Where there are both normal and deep disagreements, it is more likely to find (A) and (B).

4) So, deep disagreements offer the good news that we live in a society where (A) and (B) hold.

Let's consider the claim that without deep disagreements, it is more likely that beliefs are based on social-conditioning rather than reason and evidence. For example, suppose that in the future America is a democracy where everyone believes only in the scientific method with respect to discovering truths about the fundamental nature of reality, and that evolutionary biology and psychology provide the best account of why humans exist and how their minds work. While there are no deep disagreements over how to discover what the fundamental nature of reality is, there are still many normal disagreements, such as over challenges to the standard model of quantum mechanics, kin vs. individual selection, or massive modularity about the mind. Rather than claiming that beliefs in such a society are more likely based on social conditioning than reason and evidence, it might be more accurate to say that the scope of reason and evidence is more limited when there are only normal disagreements. For example, in such a scientific society we might not question the axioms of probability theory or the laws of nature, but we might still debate new methods for discovering truths about our universe or how best to interpret the fossil record. Moreover, even in a society where there are deep disagreements, social conditioning plays a role in the formation of one's beliefs quite early on. Social conditioning is always in play. What is up for grabs between a society that only has normal disagreements and one that also has deep disagreements is the scope of how far rational argumentation and evidence extend. When there are deep disagreements we are likely to have a society that has different moral

${ }^{4}$ This is my version of the argument that Aikin \& Talisse seem to offer on p. 80 . 
and epistemic communities, but not necessarily less social conditioning in the formation of our beliefs.

In addition, we can ask a question about tradeoffs: What is more important (C) harmony in a society with only normal disagreements or (D) that there is robust intellectual freedom and a greater likelihood, if that is actually true, that one's beliefs are based on reason and evidence as opposed to mere social conditioning?

Take as an example the authors' discussion of deep disagreements in the sense of them being absolutely deep, so deep that there is no way to resolve things.

Argument is surely possible under conditions of severely deep disagreement. What's more, when confronting an especially deep disagreement, disputants can still reason together about the depth of the disagreement (Aikin \& Talisse, 2020: 88).

When philosophers argue, I believe it would be correct to say that argument is surely possible under conditions of severely deep disagreement. But when people who neither have the professional obligation, as philosophers do, nor the time, nor the devotion to engage with others that fundamentally see them as separate but equal, it is very hard to see how productive argument for the purposes of resolving a disagreement can occur. Furthermore, this problem of disagreement can be extremely severe when we take it into the realm of meaning.

Although Tom and John were both raised in the US, Tom is highly religious and has learned all of his moral vocabulary from Bible study. John, on the other hand, is an atheist, and uses the language of political control, rather than morality. Now John and Tom get together to have a discussion on how America should be run. Morality comes up. They share their views. But we should wonder: are they even talking about the same thing? That is, when two people occupy sufficiently distinct worldviews, even when they are using the same words, for example the word, "justice", they might not mean the same thing or even come close to meaning the same thing. John and Tom might simply be talking past each other while sounding like they are talking about the same thing. More than the problem of simulated argument, we have the problem of binding and traction. When two or more parties are involved in a debate it takes more than using the same words and engaging each point made by each side to have a productive debate. Productive debate (that goes beyond merely recognizing that both parties disagree) requires agreement on at least three things: (i) the rules of debate, (ii) a set of facts, and (iii) the meaning of central terms in the debate. If we don't even mean the same thing, then what looks to be a real debate might turn out to be two groups talking past each other. While both use the word "red", one sees red, the other green. Maybe each party at least comes to see that they mean different things by key words. But what might be more useful, instead of debate, is to simply have each party present their worldview 
and positions without any argumentation between them. And the audience can decide which world they want to be a part of. Perhaps, sometimes rhetorical-patient oriented argumentation is good because two positions don't occupy a similar enough epistemic culture, and thus the best thing to do is try to win new members to one's own tribe by simply painting the other person and position as wrong. Again: is there room for legitimate rhetorical argumentation on the view of political argumentation offered by the authors?

\section{IS AD HOMINEM A FALLACY?}

Political argumentation has become uncivil to say the least. One way it has become uncivil is due to the common occurrence of ad bominem like engagements. Watch any recent presidential debate. The authors take note of this, and they characterize the fallacy in the following way:

Premise: Person $\mathrm{S}$ is in some specified way vicious.

Conclusion: We should reject the things S says.

For example, it might be claimed that $\mathrm{S}$ is a hypocrite, an alcoholic, a person with low IQ, a racist, a pervert, a neoliberal, etc. With respect to its use in political argumentation, the authors say the following:

Consider that ad hominem attacks are most frequently deployed for the sake of an audience of sympathetic onlookers. When a liberal calls his conservative interlocuter a "wing-nut," it's not for the sake of winning over the conservatives: rather, the smear is meant to serve as a cue to the liberals who might be observing the exchange. To be more precise, the ad hominem, serves to convince one's allies that one's interlocuter is vicious and unsavory, and therefore either uninterested in or incapable of civility (Aikin \& Talisse, 2020: 101).

Ad bominem is an informal fallacy pertaining to relevance. I believe this means that it is a fallacy pertaining to relevance that cannot be identified on the basis of form alone. Rather one must consider the content of what is said, the context in which it occurs, and the intentions of the speaker making the speech act. I am not confident that the authors have a completely accurate account of what some speech acts that look like ad bominem are doing in political argumentation. There are a number of cases where all that is going on is that person $A$ is bringing to light supposed facts about $B$ in order to paint $\mathrm{B}$ as bad in some direct way that pertains to character. Likewise, B is trying to do the same thing to A. Both B and A aim to defend themselves by saying that the characterization given by the other person is (i) false or (ii) taken out of context. Part of political argumentation concerns, not just policies, 
but people also. Moreover, who we want our leader to be has a lot to do with character traits we value, not just policy positions. As a consequence, some of the attacks that look like ad hominem attacks might actually turn out to be (il) legitimate attacks on a person's character. Moreover, we might ask the authors the following: Is it acceptable to draw a person's character into question when they are applying to a very high public office? And if it is, then might it be the case that not all ad bominem in form attacks are really ad bominem. Rather, they might be legitimate character queries that are necessary for healthy political argumentation? When running for president isn't a person's political character up for question as well as their prior voting record and the political policies and platform that they stand for?

\section{IS POLITICAL ARGUMENTATION SUPPOSED TO BE TOTALLY RATIONAL?}

One of the central aims of the authors' work is to articulate The Owl of Minerva Problem, an early version of which arises in the beginning of the book:

Our attempts to correct democratic practice requires creating new rules and norms for democratic citizens to follow, or perhaps reminding citizens of rules that they implicitly endorse and which we all could follow. However, partisan divisions are at present so severe that any such proposal will be received by some significant segment of the citizenry as biased against their own political allegiances; thus, our strategies of correction are transformed into tools for partisan attack. Any tools we might devise for fixing democracy will become additional instruments for its dysfunction. [...] Our understanding of democratic argumentation applies only in retrospect, because once we make that understanding public, we change the practice of democratic argumentation (Aikin \& Talisse, 2020: 4-5).

In the final chapter, they develop the full version of the problem:

In order for democracy to thrive, citizens must be able to disagree civilly, to argue well together about the social and political world they share. But in order to argue well, they must develop together a second-order vocabulary aimed at assessing arguments - a metalanguage about disagreement - that preserves the distinction between assessments of the views in dispute and assessments of the character of the reasons disputants offer in support of their respective positions. However, the metalanguage lends itself to a specific kind of abuse; once the second-order concepts make their way into argumentative practice, they can be deployed at the first-order as just additional ways of dismissing, slighting, and impugning those with whom one disagrees. This occasions a need for additional second-order concepts that can identify these new abuses. But, of course, the minting of further second-order concepts only occasions new abuses (Aikin \& Talisse, 2020: 127). 
Their assessment of the problem is severe: they see the Owl of Minerva Problem as an inevitable consequence of democracy:

Our tools for promoting civility are inevitably condemned to produce new forms of incivility, and the pathologies of public discourse are always at least one step ahead of our capacity to understand and correct them. The tragedy is inescapable (Aikin \& Talisse, 2020: 127, emphasis added).

I agree with the authors' presentation of and assessment of the problem, as well as with the importance of drawing it to the attention of scholars and the public at large. This is why their work should be read widely. There is, nevertheless, another interpretation, on which the Owl of Minerva Problem isn't as much a problem, but rather a fact about democracies that reveals that something more than rational argumentation is going on.

There are two types of disagreements. Those that are over facts and those that are over methods. When two or more parties disagree over facts alone, then what they disagree about, for example, is how many people were actually at the 2016 presidential inauguration. What they don't disagree over is what methods could and should be used in order to determine the answer. However, when two or more parties disagree over methods alone, then what they disagree about, for example, is whether counting people on a photograph of the presidential inauguration is a legitimate way to actually determine how many people were there. Moreover, when there are disagreements over methods in a democracy, that typically signals that the democracy has different epistemic cultures. For some people reading a religious text is not just a way to learn about what the religion dictates, but also a source of evidence for how the world ultimately is. For others reading a religious text at best tells us only incompletely what the religion says, and provides no evidence for how the world ultimately is.

As I noted earlier when we have a disagreement over methods it is much harder to see how we can epistemically engage each other as partners searching for the truth. If two or more parties disagree about what methods are the ones to be used, treating each other as a partner in a dance seems impossible. We can't be dancing with each other, if one person is doing the Tango and the other person is doing the Foxtrot. In most democracies there is both disagreements over facts and over methods. Sometimes one side is simply trying to win over others that are either neutral or not entrenched on the other side. They are trying to do this not by rational argumentation and evidence, but by simply painting all other alternatives as being worse than their own, and speaking about the bad character of those that occupy other positions. And they are doing this to gain power. The road to the top need not be about finding the view that is best for all. Rather, selling the view that is best only for some, 
at the cost of others, is the only way to the top. And dominance is all that is sought. We might therefore want to look for the reason behind polarization in a democracy. Is polarization happening primarily because of a difference over methods or because of a difference over facts? If it is primarily a disagreement over facts, then plausibly a democracy with the epistemic view of argumentation and the partner model of engagement is appropriate. However, if there is a substantial amount of disagreement over methods, we might diagnose the problem differently.

Consider 'status anxiety' - the anxiety over the status of a group one belongs to in a society that is comprised of various such groups. Political polarization could be a reflection of increased status anxiety. We can all tolerate a little bit of up and down in our status, but certain political ideologies threaten our status in a deep way leading to higher anxiety. This heightened anxiety could lead to groups attempting to minimize the amount of status change they undergo. It might therefore be useful to look at political argumentation as not only being about rational argumentation aimed always at getting the best view for all, but also about status (re)negotiation amongst various groups. Of course, status (re)negotiation might take place via rational arguments, but (re)negotiations cannot be advantageous to all, and so at some point rhetorical argumentation would be appropriate as opposed to epistemic argumentation.

While it is true that a democracy requires us to be political equals, it doesn't follow from our political equality that we are either (i) epistemic equals or (ii) moral equals. To portray democracy as only about rational argumentation, which is clearly better served on the epistemic-partner model of argumentation than on the rhetorical-patient model, would be inaccurate, and potentially harmful. Democracy, at least in the US, requires that individuals that are neither epistemic equals, because they don't know the same things, nor moral equals, because they don't believe in the same moral standards, must still be treated as political equals. Political equality simply means that each person's vote counts the same, and each person's contribution to the conversation deserves to be heard on the basis of the fact that everyone is governed by the decision made after deliberation. Although political equality is important, and a great advantage that democratic systems have over non-democratic ones, political equality is not sufficient to bring harmony to a society.

Democratic decision making cannot be modeled on sports competitions and sportsmanship, since what is at stake is different. We cannot expect harmony amongst citizens simply because good citizenship requires civility the way that sports competitions require good sportsmanship. We cannot make sense of the epistemic-partner model of argumentation as the whole story we should be aiming for within political argumentation in the US. We also need to consider status (re)negotiation. In order to see someone who does not hold the same belief as oneself as a partner in the search for what is best to believe, 
one must additionally believe that there are methods or mechanisms by which we can come to see a way forward.

\section{WHAT SHOULD WE GIVE UP ON?}

The authors close the book with the following:

To be clear, we conceded that democracy as it is currently practiced is a far cry from the ideal of collective self-government among political equals by means of civil disagreement. It's less clear that our failings constitute sufficient reason to jettison the idea altogether (Aikin \& Talisse, 2020: 128).

I agree that our failings don't constitute sufficient reason to jettison the idea. But the failings might constitute a sufficient reason to leave membership of one democracy and attempt to gain membership in another or form another one altogether. Let's return to the marriage analogy.

One might find themselves in a bad marriage. A marriage that was once good, but for which the parties could not have predicted that they would develop into the individuals they now are. Individuals who have too much opposition over fundamental beliefs, for example, about how to raise kids, how to do finances, or who is responsible for taking out the trash. Nevertheless, both parties might think that their fundamental disagreements are not good reasons to think that the institution of marriage is bad. Rather, they might simply think that those disagreements are good reasons to seek another marriage, one where there is more symmetry and alignment over basic ideals.

Likewise, democracy might function better when there is more alignment between those that are governed by the democracy. Aikin and Talisse's narrative on democracy makes it seem as if democratic engagement requires that citizens stay together no matter what differences arise. That is, their narrative makes me wonder: is it okay to raise the issue of whether two or more new democracies should be formed because of deep disagreements and the presence of distinct moral and epistemic cultures?

\section{BIBLIOGRAPHY}

Aikin, S. \& Talisse, R. (2020). Political argument in a polarized age: Reason and democratic life. Cambridge: Polity Press.

Vaidya, A. (2013). Epistemic responsibility and critical thinking. Metaphilosophy, 44(4), 533-556. Vaidya, A. (2015). Public philosophy: Cross-cultural and multi-disciplinary. Journal of Comparative Pbilosopby, 6(2), 35-57.

Vaidya, A. (2018). Making the case for Jain contributions to critical thinking education. Journal of World Philosophies, 3(1), 53-78. 
